\title{
Demineralized Bone Matrix (DBM) as a Bone Void Filler in Lumbar Interbody Fusion : A Prospective Pilot Study of Simultaneous DBM and Autologous Bone Grafts
}

\author{
Bum-Joon Kim, M.D., Ph.D., Se-Hoon Kim, M.D., Ph.D., Haebin Lee, B.N., Seung-Hwan Lee, M.D., Won-Hyung Kim, M.D., \\ Sung-Won Jin, M.D. \\ Department of Neurosurgery, Korea University Ansan Hospital, Ansan, Korea
}

Objective : Solid bone fusion is an essential process in spinal stabilization surgery. Recently, as several minimally invasive spinal surgeries have developed, a need of artificial bone substitutes such as demineralized bone matrix (DBM), has arisen. We investigated the in vivo bone growth rate of DBM as a bone void filler compared to a local autologous bone grafts.

Methods : From April 2014 to August 2015, 20 patients with a one or two-level spinal stenosis were included. A posterior lumbar interbody fusion using two cages and pedicle screw fixation was performed for every patient, and each cage was packed with autologous local bone and DBM. Clinical outcomes were assessed using the Numeric Rating Scale (NRS) of leg pain and back pain and the Korean Oswestry Disability Index (K-ODI). Clinical outcome parameters and range of motion (ROM) of the operated level were collected preoperatively and at 3 months, 6 months, and 1 year postoperatively. Computed tomography was performed 1 year after fusion surgery and bone growth of the autologous bone grafts and DBM were analyzed by ImageJ software.

Results : Eighteen patients completed 1 year of follow-up, including 10 men and 8 women, and the mean age was 56.4 (32-71). The operated level ranged from $L 3 / 4$ to $L 5 / S 1$. Eleven patients had single level and 7 patients had two-level repairs. The mean back pain NRS improved from 4.61 to $2.78(p=0.003)$ and the leg pain NRS improved from 6.89 to $2.39(p<0.001)$. The mean K-ODI score also improved from 27.33 to $13.83(p<0.001)$. The ROM decreased below 2.0 degrees at the 3 -month assessment, and remained less than 2 degrees through the 1 year postoperative assessment. Every local autologous bone graft and DBM packed cage showed bone bridge formation. On the quantitative analysis of bone growth, the autologous bone grafts showed significantly higher bone growth compared to DBM on both coronal and sagittal images ( $p<0.001$ and $p=0.028$, respectively). Osteoporotic patients showed less bone growth on sagittal images.

Conclusion : Though DBM alone can induce favorable bone bridging in lumbar interbody fusion, it is still inferior to autologous bone grafts. Therefore, DBM is recommended as a bone graft extender rather than bone void filler, particularly in patients with osteoporosis.

Key Words : Arthrodesis · Autograft · Bone substitutes · CT scan · Osteoporosis · Spinal fusion.

- Received : January 5, 2017 •Accepted : February 2, 2017

- Address for reprints : Se-Hoon Kim, M.D., Ph.D.

Department of Neurosurgery, Korea University Ansan Hospital, 123 Jeokgeum-ro, Danwon-gu, Ansan 15355, Korea

Tel : +82-31-412-5053, Fax : +82-31-412-5054, E-mail : sehoonkim.ns@gmail.com

This is an Open Access article distributed under the terms of the Creative Commons Attribution Non-Commercial License (http://creativecommons.org/licenses/by-nc/4.0) which permits unrestricted non-commercial use, distribution, and reproduction in any medium, provided the original work is properly cited. 


\section{INTRODUCTION}

Fusion operations are widely performed to treat lumbar spinal stenosis. The instrumentation provides stability for the first few months, and bone union, also called solid fusion, maintains stability thereafter ${ }^{11,29)}$. It is widely accepted that an autologous bone graft using an iliac bone harvest is the gold standard in spinal fusion because it can provide all three essential properties (osteogenesis, osteoinduction, and osteoconduction) required for bone fusion ${ }^{6,8,18)}$. However, the major disadvantage of this procedure is the unavoidable donor site morbidity ${ }^{8,18)}$. Moreover, more recent minimally invasive surgeries, such as direct lumbar interbody fusion (DLIF), make it difficult to obtain adequate amounts of local autologous bone chips.

There have been many efforts to enhance fusion rate, with the hope of eventually entirely replacing the autologous bone grafts $^{211}$. One such development is a bone graft substitute, such as demineralized bone matrix (DBM), which was found to have fair osteoinductive and osteoconductive properties in previous studies ${ }^{4,25,26}$. Numerous studies have reported superior or equivalent outcomes of DBM as a bone graft extender compared to autologous bone grafts alone ${ }^{1,17}$. However, evidence regarding whether DBM is sufficient as bone void filler in lumbar spinal fusion is still lacking.

Many host factors, including osteoporosis, hormone therapy, medications, nutrition and smoking status, can influence the bone graft, and the surgeon's technique including the preparation of the fusion bed, instrumentation, and manipulation of bone graft, can affect the fusion rate ${ }^{2,18}$. An in vivo study performed in the same bone growth environment is required to determine the true fusion rate of DBM as bone void filler.

We present our prospective study of patients who underwent lumbar interbody fusion using both autologous bone grafts and DBM as bone void filler.

\section{MATERIALS AND METHODS}

\section{Patient population}

From April 2014 to August 2015, twenty patients with spinal stenosis were included in this prospective study. Patients between ages 20 and 75 who were diagnosed with lumbar spinal stenosis or spondylolisthesis and eventually required a one or two-level fusion surgery were included in the study. The exclusion criteria were as follows : having undergone revision surgery, trauma, compression fracture, presence of malignancy, or infectious disease. Clinical and radiographic data were collected in accordance with the regulations of the institutional review board at Korea University Ansan Hospital.

\section{Clinical and radiographic data acquisition}

Clinical outcomes were assessed using the Numeric Rating Scale (NRS) of leg and back pain and the Korean Oswestry Disability Index (K-ODI ${ }^{12}$. To assess for successful fusion, the range of motion (ROM) of the operated segment was measured using the cobb angle from the lateral flexion-extension simple radiographs acquired at the pre-operative assessment and at 3 months, 6 months, and 1 year postoperatively. We performed dual-energy x-ray absorptiometry (DEXA) scans to see if the patient's bone density influenced fusion.

Computed tomography (CT) was performed at 1 year after fusion surgery to assess the amount of bone bridging at the graft sites. To quantify and objectively compare the amount of bone growth, we cropped the operated intervertebral space from the coronal and sagittal CT images, visualizing the center portion of the polyetheretherketone (PEEK) cage. From the coronal image, after inverting the white and black colors, we performed densitometric analysis on the white vertical bandlike bone bridge inside the cages (Fig. 1A), using ImageJ 1.50i software (W. Rasband, National Institutes of Health, Bethesda, MD, USA; available at http://rsb.info.nih.gov/ii/). From the sagittal CT images, two mid-cage (autologous bone grafts and DBM) slices were collected from each patient (Fig. 1B). The white bone growth area inside the cage was measured by pixel calculation using ImageJ software.

\section{Operative technique}

During the operation, specifically during conventional posterior lumbar interbody fusion (PLIF), PEEK cages were used for interbody fusion to visualize bone growth on radiographs. After subtotal laminectomy and bilateral medial facetectomies, the local autologous bone chips were collected and the cancellous portion was used to fill the PEEK cage on the right side of the patient. After insertion of the cage on the right side, the remnant autologous bone chips were placed between the cages. Then, another PEEK cage filled with putty-type DBM (Bonfuse ${ }^{\bowtie}$ CGBio, Seoul, Korea) was inserted into the left in- 

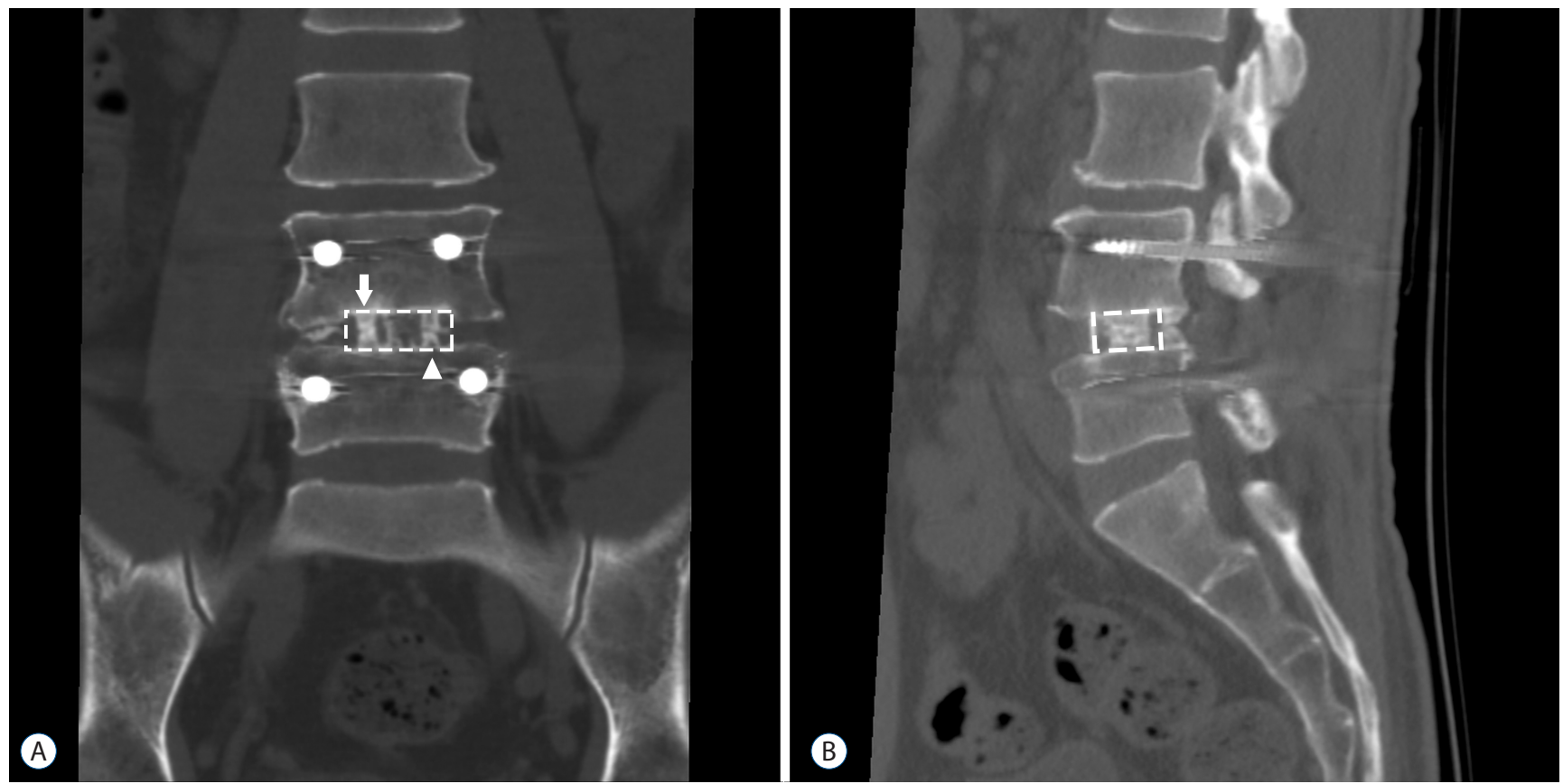

Fig. 1. Representative computed tomography of patient included in this study. A : A coronal view shows hyperdense bony bridges connecting the cranial and caudal vertebral bodies. The polyetheretherketone (PEEK) cage on the right side is packed with autologous bone grafts (arrow) and the cage on the left side is packed with demineralized bone matrix (arrowhead). The dotted rectangular area was used for densitometric analysis of the bone bridges. B : A sagittal view of each mid-cage section shows the bone growth area occupying the cage. The graft area inside the PEEK cage (dotted rectangle) was cut and the number of pixels of the white colored areas was calculated.

tervertebral space. For every patient, pedicle screws were inserted and compression against the interbody cages followed.

\section{Statistical analysis}

Statistical analyses were performed with IBM SPSS statistics 20.0 software (IBM Corp., Armonk, NY, USA). The comparison between preoperative and postoperative parameters and between DBM and autologous bone grafts parameters were performed using paired t-tests, except for the assessment of the pixels from the sagittal view, which were compared using Wilcoxon signed rank test due to its lack of a normal distribution. The existence of an association among the numerical parameters was assessed by Pearson correlation and partial correlation analysis.

\section{RESULTS}

Of the initial 20 patients, 2 patients were excluded because of lack of 1-year follow-up. The remaining 18 patients consisted of 10 men and 8 women with a mean age of 56.4 (32-71).
The operated level ranged from L3/4 to L5/S1. Eleven patients had single-level and 7 patients had two-level repairs. As shown in Fig. 2, all the mean values of the NRS and K-ODI clinical outcome improved at the 1-year postoperative assessment. The mean value of the back pain NRS improved from 4.61 to $2.78(p=0.003)$, and that of leg pain NRS improved from 6.89 to $2.39(p<0.001)$. The mean K-ODI score also improved from 27.33 to $13.83(p<0.001)$. Age, sex, and the operated level were not significantly correlated with clinical outcomes.

The measured ROM of the operated segment dramatically decreased in all patients, from 7.75 degrees to 1.93 degrees $(p<0.001)$. The ROM decreased below 2.0 degrees at the 3 -month assessment, and remained less than 2 degrees through the 1 year postoperative assessment (Fig. 2D).

On the follow up CT scan at 1-year postoperative assessment, bone bridge formation inside the cage was observed in every patient regardless of whether the graft material was autologous bone or DBM (Fig. 1). On the quantitative comparison of bone growth, autologous bone graft showed a higher density of bone bridges on the coronal CT view (Fig. 3A, $p<0.001)$ and a larger area on the sagittal CT view compared 


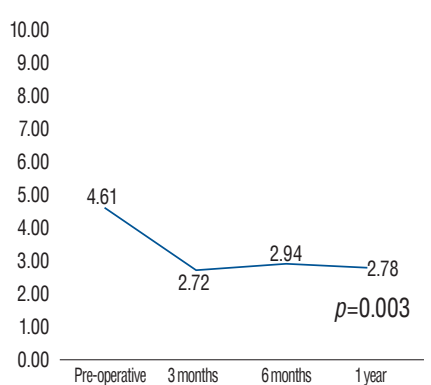

(A)

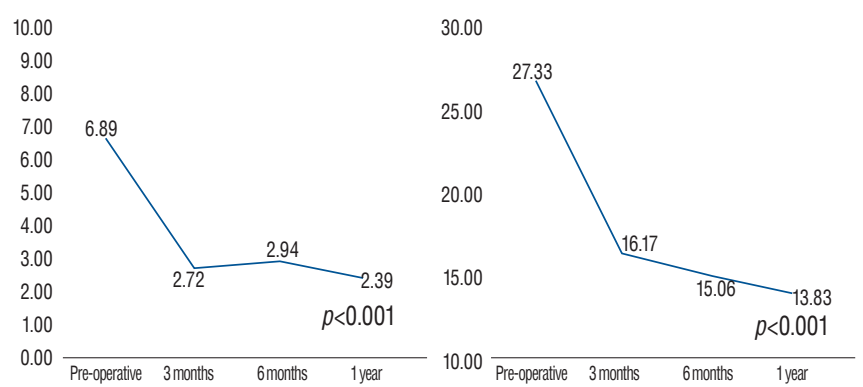

(C)

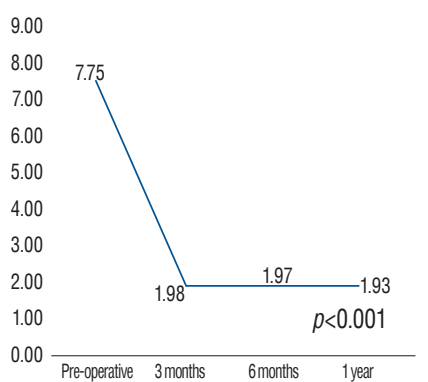

(D)

Fig. 2. Line chart of clinical outcomes and range of motion (ROM). A : Numeric rating scale (NRS) of back pain. B : NRS of leg pain. C : Korean Oswestry Disability Index (K-ODI ). D : ROM at the operated level.

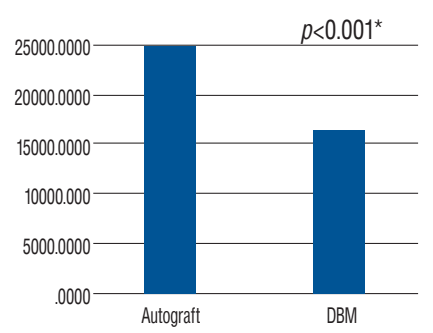

(A)

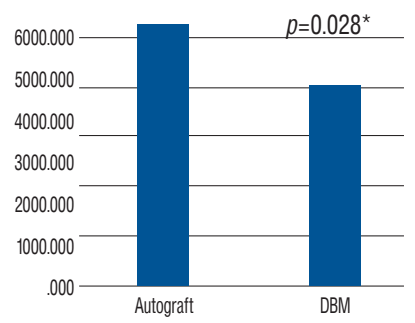

(B)

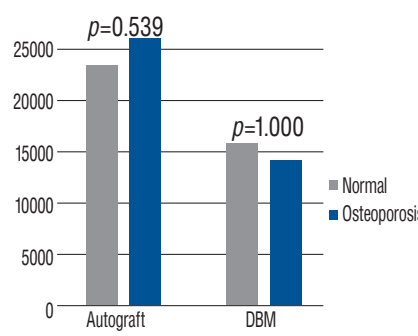

(C)

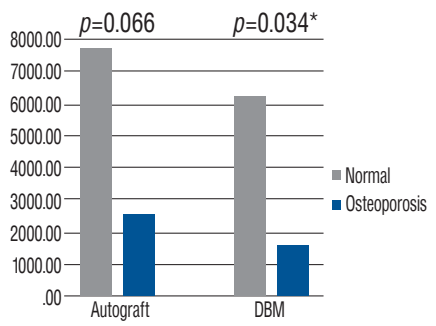

(D)

Fig. 3. Histogram of comparative analysis of bone growth between autologous bone grafts and demineralized bone matrix (DBM). A : Densitometric analysis of bone growth on coronal view. B : Measurement of bone growth area on sagittal view. C : Comparison of bone growth with osteoporosis group on coronal view. D: Comparison of bone growth with osteoporosis group on sagittal view. ${ }^{*}$ Indicate statistically significant difference with $p<0.05$.

to DBM (Fig. 3B, $p=0.028$ ).

For bone density, the mean T-score was -0.96 . With adjustment for age, which demonstrated a negative correlation with T-score $(p=0.005)$, the partial correlation analysis did not show an association between $\mathrm{T}$-score and bone growth. There were four osteoporotic patients whose T-score was under -2.5. Though densitometric analysis on coronal CT views did not show a significant difference based on osteoporosis (Fig. 3C), on sagittal CT views, both the autologous bone and the DBM side of osteoporotic patients showed much smaller bone growth areas than those of normal patients ( $1 / 4$ and $1 / 3$, $p=0.066$ and $p=0.034$, respectively) (Fig. 3D).

\section{DISCUSSION}

DBM is an acid extraction product of cadaver bone, which was first developed by Marshall Urist in $1965^{27}$. While most bone graft substitutes and synthetics show osteoconductive property rather than osteoinductive property, DBM is known to have osteoinductive properties as well ${ }^{8,921)}$. Though its demineralization process remove cells and structural strength from the cadaver bone, its remaining proteinaceous components enhance the bioactivity ${ }^{6,18,21,27)}$. DBM is widely used in recent spine surgeries, and the most popular type of DBM graft is the putty type, which has the viscosity to prevent wash-out by irrigation ${ }^{9)}$.

In lumbar posterolateral fusion, Cammisa et al. suggested that DBM can reduce the quantity of autologous bone grafts harvest and Morone et al. suggested that DBM was more effective when it was applied as a bone graft extender in posterolateral fusion ${ }^{3,19)}$. Different from posterolateral fusion, which, according to Wolff's Law ${ }^{5}$, cannot provide enough compression to the bone graft, we expect that DBM can be a promising alternative for autologous bone grafts in interbody fusion. Kim et al. reported similar fusion rates for DBM compared to autologous bone grafts as a bone void filler in lumbar interbody fusion cases ${ }^{13}$. In this study, we applied autologous bone grafts and DBM simultaneously and separately in each patient to control for host and surgeon factors. We 
found that the natural bone growth of autologous bone grafts using local bone was superior to that of DBM. The bone growth of the DBM side was $65 \%$ of the autologous bone grafts on densitometry and $77 \%$ in area by pixels (Fig. $3 \mathrm{~A}, \mathrm{~B})$. On the other hand, though it was not included in the analysis, even the autologous bone grafts, between the cages, showed favorable bone growth in most patients. This finding implies that spine surgeons can gain additional fusion beds by packing remnant bone chips to the disc spaces outside the cages.

A CT scan has superior sensitivity to show bone growth in the graft area compared to a simple radiograph ${ }^{15)}$. Particularly, three-dimensional (3D) helical CT is very useful for visualizing the growing bone bridges ${ }^{15)}$. In this study, we collected coronal and sagittal images of the patients to identify whether the cranial and caudal endplate is connected by vertical bone bridges (Fig. 1). In the interpretation of fusion using the CT scans, most previous studies have focused on the presence and continuity of bone bridges ${ }^{7,13,24)}$. However, a simple classification based on presence or absence may have some limitations. Clinicians usually prefer to limit CT scans because of radiation exposure. When the CT scan follow-up is done too early or too late, it is difficult to reveal the different fusion rates between graft materials. In our series, on the one-year follow-up CT, 9 of 18 patients showed continuous bone bridges. Moreover, the remaining 9 patients still showed gaps between the cranial and caudal bone bridges. Among the 9 patients with continuous bridging, 3 patients showed bilateral bridging, 4 patients showed bridging only on the autologous bone graft side, and 2 patients had bridging on the DBM side. We could not find significant differences regarding the "continuity of bone bridges" between autologous bone grafts and DBM. However, the calculated pixels of the bone bridge were significantly higher in autologous bone grafts (Fig. 3).

Though some authors reported that a flexion-extension radiograph is a simple, favorable method to determine whether the patient's operated level achieved solid fusion ${ }^{7)}$, it is limited in its ability to identify a nonunion that has subtle motion ${ }^{15)}$. Larsen et al. demonstrated that the stability of the pedicle screw can restrict meaningful motion even in patients with pseudarthrosis ${ }^{16)}$. In our series, even in patients who showed gaps between the bridges, the ROM was maintained below 2 degrees during the 1-year follow up period (Fig. 2D).
Assuming that modern spinal devices prevent obvious motion even in cases of nonunion, we suggest that a multidisciplinary assessment, including clinical examination, flexionextension radiograph, and CT scan, is required to interpret "solid fusion" in lumbar interbody fusion.

A PEEK cage, due to its less stiff Young's modulus, is known to have a superior load sharing effect than titanium cages ${ }^{22,28)}$. However, it has an inferior osseointegration ability ${ }^{20,23)}$. Therefore, solid fusion of the graft packed into the cage is mandatory to prevent pseudarthrosis. PEEK cages cause less artifact changes on a radiograph, so the radiolucent characteristics of the PEEK cage are very useful to assess the pattern of bone growth inside the cages ${ }^{7,22}$. In this study, we could easily identify the extent and continuity of the bone bridges inside the PEEK cages (Fig. 1).

Based on previous studies, osteoporosis impairs the bone healing process after fractures and can result in lower fusion rates in spine surgeries ${ }^{10,14,18}$. In this study, patients with osteoporosis showed less bone formation in both autologous bone grafts and DBM (Fig. 3D) compared to patients without osteoporosis. The bone growth area in the DBM packed cage was significantly smaller $(1: 4)$ in osteoporosis patients (Fig. 3D). Though none of the 4 osteoporotic patients showed pseudarthrosis during 1-year follow up, we recommend autologous bone grafts for patients with osteoporosis, and harvesting of the iliac crest should be considered as well.

The strength of our study is that we observed the simultaneous natural bone growth rate of autologous bone grafts and DBM in vivo and in same environment, and performed analysis using enumerated data obtained from CT images and image calculation software. However, there are some limitations. Some comparative analyses failed to reach statistical significance due to the small population size, particularly in the osteoporosis cohort. Another limitation was that our patients received autologous bone grafts and DBM at the same time. Therefore, we could not evaluate pure clinical outcomes of DBM alone. Our follow-up period was 1 year, which may have underestimated the development of pseudarthrosis and instrument failure. Patients who showed incomplete bridging during the first year require further follow up to determine whether they will develop solid fusion or pseudarthrosis. 


\section{CONCLUSION}

Both local autologous bone grafts and DBM can induce favorable bone growth independently in lumbar interbody fusion. If it is available, DBM should be used as an autologous bone graft extender. In situations where access to autologous bone grafts is limited, such as minimally invasive surgery, DBM can be used as bone void filler. In osteoporotic patients, DBM is recommended as a graft extender, not bone void filler, and an iliac crest harvest should be considered.

\section{- Acknowledgements}

This research was supported by a research grant from CGBio Co. Ltd. (Korea).

\section{References}

1. An HS, Simpson JM, Glover JM, Stephany J : Comparison between allograft plus demineralized bone matrix versus autograft in anterior cervical fusion. A prospective multicenter study. Spine (Phila Pa 1976) 20 : 2211-2216, 1995

2. Boden SD, Schimandle $J H$ : Biologic enhancement of spinal fusion. Spine (Phila Pa 1976) 20(24 Suppl) : 113S-123S, 1995

3. Cammisa FP Jr, Lowery G, Garfin SR, Geisler FH, Klara PM, McGuire RA, et al. : Two-year fusion rate equivalency between Grafton DBM gel and autograft in posterolateral spine fusion : a prospective controlled trial employing a side-by-side comparison in the same patient. Spine (Phila Pa 1976) $29: 660-666,2004$

4. Chalmers J, Gray D, Rush J : Observations on the induction of bone in soft tissues. J Bone Joint Surg Br 57 : 36-45, 1975

5. Farrokhi MR, Rahmanian A, Masoudi MS : Posterolateral versus posterior interbody fusion in isthmic spondylolisthesis. J Neurotrauma 29 : 1567-1573, 2012

6. Finkemeier CG : Bone-grafting and bone-graft substitutes. J Bone Joint Surg Am 84 : 454-464, 2002

7. Fogel GR, Toohey JS, Neidre A, Brantigan JW : Fusion assessment of posterior lumbar interbody fusion using radiolucent cages : X-ray films and helical computed tomography scans compared with surgical exploration of fusion. Spine J 8 : 570-577, 2008

8. Giannoudis PV, Dinopoulos H, Tsiridis E : Bone substitutes : an update. Injury 36 Suppl 3 : S20-S27, 2005

9. Gruskin E, Doll BA, Futrell FW, Schmitz JP, Hollinger JO : Demineralized bone matrix in bone repair : history and use. Adv Drug Deliv Rev 64 : 1063-1077, 2012

10. He YX, Zhang G, Pan XH, Liu Z, Zheng LZ, Chan CW, et al. : Impaired bone healing pattern in mice with ovariectomy-induced osteoporosis : A drill-hole defect model. Bone 48 : 1388-1400, 2011
11. Kanayama M, Cunningham BW, Weis JC, Parker LM, Kaneda K, McAfee $P C$ : The effects of rigid spinal instrumentation and solid bony fusion on spinal kinematics : a posterolateral spinal arthrodesis model. Spine (Phila Pa 1976) 23 : 767-773, 1998

12. Kim DY, Lee SH, Lee HY, Lee HJ, Chang SB, Chung SK, et al. : Validation of the Korean version of the oswestry disability index. Spine (Phila Pa 1976) 30 : E123-E127, 2005

13. Kim DH, Lee N, Shin DA, Yi S, Kim KN, Ha Y : Matched Comparison of Fusion Rates between Hydroxyapatite Demineralized Bone Matrix and Autograft in Lumbar Interbody Fusion. J Korean Neurosurg Soc 59 : 363-367, 2016

14. Kubo T, Shiga $T$, Hashimoto J, Yoshioka M, Honjo $H$, Urabe $M$, et al. : Osteoporosis influences the late period of fracture healing in a rat model prepared by ovariectomy and low calcium diet. J Steroid Biochem Mol Biol 68 : 197-202, 1999

15. Lang P, Genant HK, Chafetz N, Steiger P, Morris JM : Three-dimensional computed tomography and multiplanar reformations in the assessment of pseudarthrosis in posterior lumbar fusion patients. Spine (Phila Pa 1976) $13: 69-75,1988$

16. Larsen JM, Rimoldi RL, Capen DA, Nelson RW, Nagelberg S, Thomas JC $\mathrm{Jr}$ : Assessment of pseudarthrosis in pedicle screw fusion : a prospective study comparing plain radiographs, flexion/extension radiographs, CT scanning, and bone scintigraphy with operative findings. J Spinal Disord 9 : 117-120, 1996

17. Lee KJ, Roper JG, Wang JC : Demineralized bone matrix and spinal arthrodesis. Spine J 5(6 Suppl) : S217-S223, 2005

18. Lind $M$, Bünger $C$ : Factors stimulating bone formation. Eur Spine J 10 Suppl 2 : S102-S109, 2001

19. Morone MA, Boden SD : Experimental posterolateral lumbar spinal fusion with a demineralized bone matrix gel. Spine (Phila Pa 1976) 23 : 159-167, 1998

20. Najeeb S, Khurshid Z, Zohaib S, Zafar MS : Bioactivity and osseointegration of PEEK are inferior to those of titanium-a systematic review. J Oral Implantol 42 : 512-516, 2016

21. Place ES, Evans ND, Stevens MM : Complexity in biomaterials for tissue engineering. Nat Mater 8 : 457-470, 2009

22. Ponnappan RK, Serhan H, Zarda B, Patel R, Albert T, Vaccaro AR : Biomechanical evaluation and comparison of polyetheretherketone rod system to traditional titanium rod fixation. Spine J 9 : 263-267, 2009

23. Sagomonyants KB, Jarman-Smith ML, Devine JN, Aronow MS, Gronowicz GA : The in vitro response of human osteoblasts to polyetheretherketone (PEEK) substrates compared to commercially pure titanium. Biomaterials 29 : 1563-1572, 2008

24. Santos ER, Goss DG, Morcom RK, Fraser RD : Radiologic assessment of interbody fusion using carbon fiber cages. Spine (Phila Pa 1976) 28 : 997-1001, 2003

25. Torricelli $P$, Fini $M$, Giavaresi $G$, Giardino R : In vitro osteoinduction of demineralized bone. Artif Cells Blood Substit Immobil Biotechnol $26: 309-315,1998$

26. Torricelli P, Fini M, Rocca M, Giavaresi G, Giardino R : Xenogenic demineralized bone matrix : osteoinduction and influence of associated skeletal 
DBM vs Autologous Bone Grafts as a Bone Void Filler in PLIF | Kim BJ, et al.

defects in heterotopic bone formation in rats. Int Orthop 23 : 178-181, 1999

27. Urist MR : Bone : formation by autoinduction. Science $150: 893-899$, 1965

28. Vadapalli S, Sairyo K, Goel VK, Robon M, Biyani A, Khandha A, et al. :
Biomechanical rationale for using polyetheretherketone (PEEK) spacers for lumbar interbody fusion-a finite element study. Spine (Phila Pa 1976) 31 : E992-E998, 2006

29. Young PM, Berquist TH, Bancroft LW, Peterson JJ : Complications of spinal instrumentation. Radiographics 27 : 775-789, 2007 